\title{
BISSEGMENTECTOMIA CENTRAL INFERIOR (S4B+S5) PARA O TRATAMENTO DO CARCINOMA INVASOR DA VESÍCULA BILIAR: revisão de sete casos operados
}

\author{
Sergio Renato Pais COSTA, Sergio Henrique Couto HORTA, Marcelo José MIOTTO, \\ Maurício Campanelli COSTAS, Carlos Alberto GODINHO e Alexandre Cruz HENRIQUES
}

RESUMO - Racional - A despeito de sua raridade, o câncer da vesícula biliar é uma neoplasia agressiva com péssimo prognóstico. A melhor ressecção oncológica permanece sendo a hepatectomia direita ampliada para o segmento IV; no entanto a bissegmentectomia IV-V tem se tornado uma alternativa interessante, pela maior preservação do parênquima hepático. Objetivo - Reportar os resultados precoces e tardios com a bissegmentectomia IV-V no carcinoma da vesícula biliar. Métodos - É apresentada uma série de sete doentes (seis mulheres e um homem) com carcinoma invasivo submetidos a bissegmentectomia IV-V no Serviço de Cirurgia Geral do Hospital de Ensino - Faculdade de Medicina do ABC, Santo André, SP. O estudo foi realizado no período de 2002 a 2006. A idade dos pacientes variou de 52 a 72 anos. O diagnóstico foi pré-operatório (radiológico) em cinco doentes, todos confirmados por exame de congelação intra-operatório. Em dois doentes foi dado pós-operatório de colecistectomia aberta. Resultados - O tempo cirúrgico variou de 180 a 340 minutos. O sangramento intra-operatório variou de 200 a $1500 \mathrm{~mL}$. Houve duas complicações maiores. A mortalidade foi nula. Seis doentes não apresentaram recurrência entre 3 a 30 meses de seguimento. Conclusão - A bissegmentectomia IV-V pode ser uma alternativa cirúrgica curativa para o tratamento do câncer de vesícula biliar. Esse procedimento apresenta morbidade e mortalidade aceitáveis.

DESCRITORES - Neoplasias da vesícula biliar. Carcinoma, cirurgia. Hepatectomia.

\section{INTRODUÇÃO}

O carcinoma da vesícula biliar foi primariamente descrito por Maximilian Destoll em $1777^{(6)}$. No entanto, coube a Keen (citado por SHEINFIELD ${ }^{(32)}$ ) realizar a primeira ressecção hepática para o tratamento dessa neoplasia. O carcinoma da vesícula biliar é o tumor maligno mais comum da via biliar e o sexto mais freqüentemente encontrado no trato digestório. Estimase que corresponda a aproximadamente $8,4 \%$ dos casos de tumores hepatobiliares diagnosticados nos Estados Unidos da América durante o biênio 1994-1995(8).

A despeito da melhora dos exames de imagem e do suporte pós-operatório aliado a maior agressividade cirúrgica, a sobrevida global do carcinoma da vesícula biliar permanece baixa, conseqüência provável de sua natureza agressiva que apresenta extensa disseminação linfática e peritonial de maneira precoce. Desta maneira, excetuando-se os casos incidentais de tumores Tis ou T1a (TNM) operados primariamente por doença benigna sintomática (colelitíase ou pólipo), a maioria dos casos se apresenta em estádios avançados, com escassa chance de cura. Falhas para o seu tratamento precoce decorrem, principalmente, do seu curso clínico insidioso, que é invariavelmente confundido com doenças benignas de menor importância clínica. Por conseguinte, o diagnóstico é freqüentemente delegado para uma fase tardia da doença. Sintomas exuberantes como icterícia, massa palpável, alteração do hábito intestinal e ascite podem representar doença avançada com baixo índice de curabilidade. Devido a essa nefasta história natural, uma atitude niilista baseada, principalmente, nos maus resultados de seu tratamento cirúrgico fez com que se diminuísse a indicação das cirurgias curativas no passado. Destarte, durante esse período histórico, a abordagem cirúrgica ficou quase que exclusivamente direcionada a um aspecto meramente paliativo. Contudo, mais recentemente, baseado em melhores resultados, principalmente da escola japonesa (com ressecções hepáticas combinadas com linfadenectomias regionais ou alargadas) houve ressurgimento do tratamento cirúrgico curativo dessa neoplasia. Para os tumores superficiais Tis, T1a ou até

Serviço de Cirurgia Geral, Hospital de Ensino, Disciplina de Cirurgia do Aparelho Digestivo, Faculdade de Medicina do ABC, Santo André, SP, Brasil. 
mesmo T1b para alguns autores, a colecistectomia simples parece ser adequada, não se necessitando de ressecção ampliada (hepática) ou linfadenectomia hilar. Contudo, para estádios mais avançados (T2 ou mais), a maioria dos estudos sugere que algum tipo de ressecção hepática complementada com linfadenectomia hilar seja incluída para o seu tratamento ${ }^{(14,20,21,23,25,30,31,39)}$.

Em que pese uma melhoria substancial dos resultados cirúrgicos recentes, dúvidas ainda pairam em relação ao melhor tipo de ressecção hepática. Contudo, é sabido que dois objetivos principais são paralelamente importantes para os bons resultados de uma cirurgia curativa nessa neoplasia: 1) a margem hepática deve ser livre de neoplasia, 2) a linfadenectomia regional deve ser adequada. A ressecção dos segmentos IV-B e V, também denominada bissegmentectomia central inferior $(\mathrm{S} 4 \mathrm{~b}+\mathrm{S} 5)$, a hepatectomia central (ressecção dos segmentos IV, V e VIII) e a hepatectomia direita ampliada para o segmento IV, têm sido os tipos de ressecção mais comumente aceitos e permanecem como condutas terapêuticas de eleição para o tratamento do carcinoma invasor da vesícula biliar nos estádios $\mathrm{T} 1 \mathrm{~b}$ até $\mathrm{T} 3^{(21,39)}$. A bissegmentectomia central inferior apresenta como principal vantagem, a maior conservação do parênquima hepático, minimizando desta maneira, a insuficiência hepática pós-operatória. Essa complicação carrega alta mortalidade e tem sido mais freqüentemente observada após hepatectomia direita ampliada $^{(11,19,20,21,28,37)}$.

Com objetivo de descrever os resultados precoces e tardios da bissegmentectomia central inferior para o tratamento do carcinoma invasivo da vesícula biliar, relata-se uma série de sete casos de carcinoma de vesícula biliar submetidos a bissegmentectomia central inferior com intuito curativo.

\section{MÉTODOS}

Ao todo 15 pacientes com câncer de vesícula foram tratados no Serviço de Cirurgia Geral do Hospital de Ensino da Faculdade de Medicina do ABC, Santo André, SP, no período de setembro de 2002 a setembro de 2006. Desses 15 pacientes, 7 tinham cânceres irressecáveis e foram tratados paliativamente. Dos oito restantes, um foi submetido a hepatectomia direita ampliada para o segmento IV, enquanto os sete restantes foram submetidos a bissegmentectomia central inferior $(\mathrm{S} 4 \mathrm{~B}+\mathrm{S} 5)$.

Seis pacientes eram do sexo feminino e um masculino. A idade variou de 52 a 72 anos com média etária de 66 anos. Em relação à etnia, todos os sete eram caucasianos. A respeito de enfermidades associadas, dois apresentavam hipertensão arterial sistêmica (HAS) enquanto outros dois apresentavam, além da
HAS, diabetes mellitus tipo II (DM). Por fim, um doente era etilista crônico e consumia cerca de $500 \mathrm{~mL}$ de destilado por dia, pelo período aproximado de 30 anos.

Todos apresentavam algum tipo de sintoma, que foram: cólica biliar $(n=4)$, dispepsia $(n=1)$, colecistite aguda $(\mathrm{n}=1)$ e icterícia obstrutiva $(\mathrm{n}=1)$. Este último doente apresentou quadro de icterícia obstrutiva por coledocolitíase, cuja resolução foi endoscópica (papilotomia endoscópica), Portanto, foi operado posteriormente já na ausência de icterícia. Quatro apresentavam perda de peso significativa ( $10 \%$ do peso) e nenhum apresentava icterícia ou massa palpável no período pré-operatório. Essas características demográficas e clínicas estão demonstradas na Tabela 1.

Exceto dois pacientes cujo diagnóstico foi pós-operatório, todos os demais apresentavam exame de imagem suspeito de neoplasia de vesícula biliar. Foi realizada ultra-sonografia (US) total de abdome em todos os pacientes. Todos apresentavam cálculos biliares, cujo número variou de 2 a 10, enquanto o diâmetro variou de 3 a $40 \mathrm{~mm}$. Seis pacientes, exceto aquele operado em urgência, realizaram tomografia computadorizada (TC) de abdome no período pré-operatório, e em dois foi realizada ressonância magnética (RNM) de abdome. Foi observada alteração radiológica em seis (US e/ou TC): lesão heterogênea, vegetante-fungóide em dois (Figura 1), espessamento focal e calcificado em um, espessamento difuso em um e massa heterogênea ocupando totalmente a fossa vesicular em dois. O paciente com icterícia prévia por coledocolitíase não

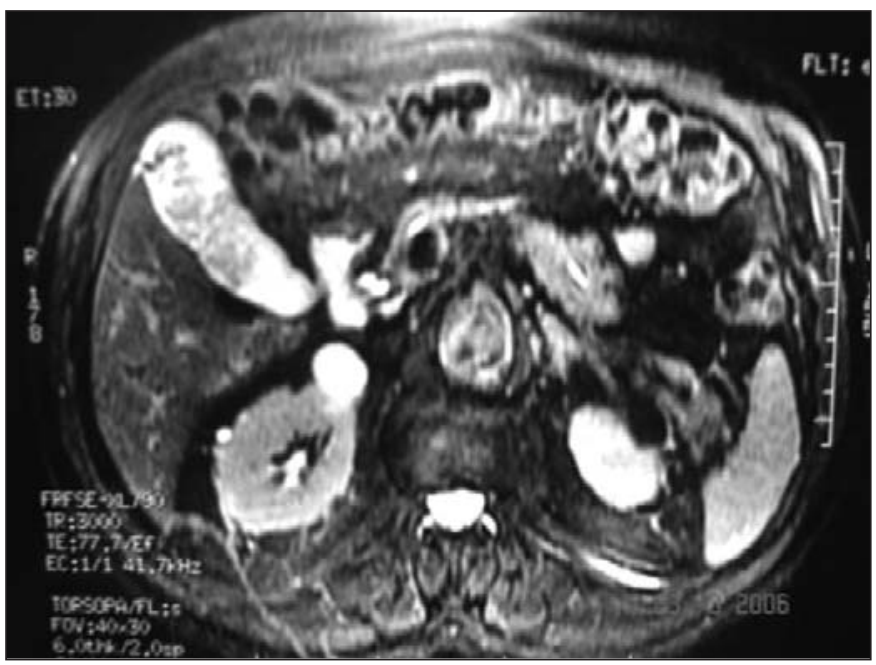

FIGURA 1. RNM - Lesão heterogênea no interior da vesícula

TABELA 1. Distribuição das características clínicas e epidemiológicas

\begin{tabular}{|c|c|c|c|c|c|c|c|}
\hline Característica & Caso 1 & Caso 2 & Caso 3 & Caso 4 & Caso 5 & Caso 6 & Caso 7 \\
\hline Sexo & feminino & feminino & feminino & feminino & feminino & feminino & masculino \\
\hline Idade & 52 & 72 & 68 & 70 & 66 & 72 & 68 \\
\hline Etnia & branca & branca & branca & branca & branca & branca & branca \\
\hline Doenças associadas & etilismo & HAS & HAS & HAS+DMII & - & HAS & _- \\
\hline Sintomas & $\begin{array}{c}\text { cólica biliar } \\
\text { emagrecimento }\end{array}$ & $\begin{array}{c}\text { cólica biliar } \\
\text { emagrecimento }\end{array}$ & $\begin{array}{c}\text { cólica biliar } \\
\text { emagrecimento }\end{array}$ & $\begin{array}{l}\text { cólica biliar } \\
\text { emagrecimento }\end{array}$ & dispepsia & $\begin{array}{c}\text { icterícia } \\
\text { obstrutiva }\end{array}$ & $\begin{array}{c}\text { colecistite } \\
\text { aguda }\end{array}$ \\
\hline
\end{tabular}


apresentava qualquer alteração na vesícula biliar tanto à US quanto à TC, exceto os cálculos biliares e a dilatação das vias biliares. Em relação à localização anatômica das lesões foram encontradas: lesão no fundo $(\mathrm{n}=4)$, no corpo $(\mathrm{n}=2)$ e difusa $(n=1)$. Foi possível a determinação da dimensão do tumor em cinco pacientes, nos exames pré-operatórios realizados. O tamanho das lesões variou de $2 \mathrm{~cm}$ a $6 \mathrm{~cm}$, com média de $3,6 \mathrm{~cm}$. Quanto ao estádio radiológico pré-operatório, dois pacientes eram T2 e três T3. Os achados radiológicos estão demonstrados na Tabela 2.

Em cinco pacientes, além dos exames radiológicos, foram dosados no período pré-operatório, os níveis de marcadores tumorais antígeno carcinoembrionário (CEA) e CA 19,9. O nível de CEA variou de 2,5 a $500 \mathrm{ng} / \mathrm{mL}$, enquanto o de CA 19,9 variou de 28 a $325 \mathrm{ng} / \mathrm{mL}$. Quando considerados os níveis de dosagem normais, quatro dos cinco apresentavam tanto CEA quanto CA 19,9 elevados. Em relação aos demais exames bioquímicos, apenas dois pacientes apresentavam ligeira elevação de fosfatase alcalina e bilirrubinas no período préoperatório. Todos pacientes apresentavam níveis normais de hemoglobina, hematócrito e albumina. Esses achados podem ser observados na Tabela 3.

Todos pacientes tiveram o tumor diagnosticado por exame histológico: cinco deles no intra-operatório, por meio de exame de congelação e em dois pacientes, o diagnóstico foi no período pós-operatório, com a avaliação histológica do espécime cirúrgico (após colecistectomia aberta).

A tática operatória que tem sido padronizada no Serviço de Cirurgia Geral do Hospital de Ensino para as lesões suspeitas de neoplasia de vesícula biliar, consiste em: incisão subcostal direita ampla, com ou sem prolongamento à esquerda, fixação de compressas para proteção e minucioso inventário da cavidade, avaliando de forma rigorosa as vias de disseminação peritonial, hepática e linfonodal (N2-N3). Qualquer indício de disseminação, o tecido é prontamente biopsiado e o material enviado para análise histológica por exame de congelação. Se positivo, a ressecção é interrompida. Não havendo disseminação, é realizada primariamente a colecistectomia. A vesícula é, em seguida, aberta no sentido longitudinal, marcando-se o ducto cístico (Figura 2) e, por final, o material é enviado para exame de congelação. Uma vez positivo para neoplasia, a cirurgia é complementada com a ressecção dos segmentos IV-B e V (Figura 3). Nos casos em que o tumor invade propriamente o parênquima hepático, a cirurgia é em monobloco e consiste na ressecção da vesícula biliar em conjunto com os segmentos IV-B e V (Figura 4). Esse tipo de cirurgia tem sido reservado somente para os casos de tumores limitados ao fundo ou corpo da vesícula e sem invasão hilar. Caso contrário, é prontamente realizada uma hepatectomia direita ampliada para o segmento IV (se o volume do lobo esquerdo remanescente for maior que $25 \%$ ).

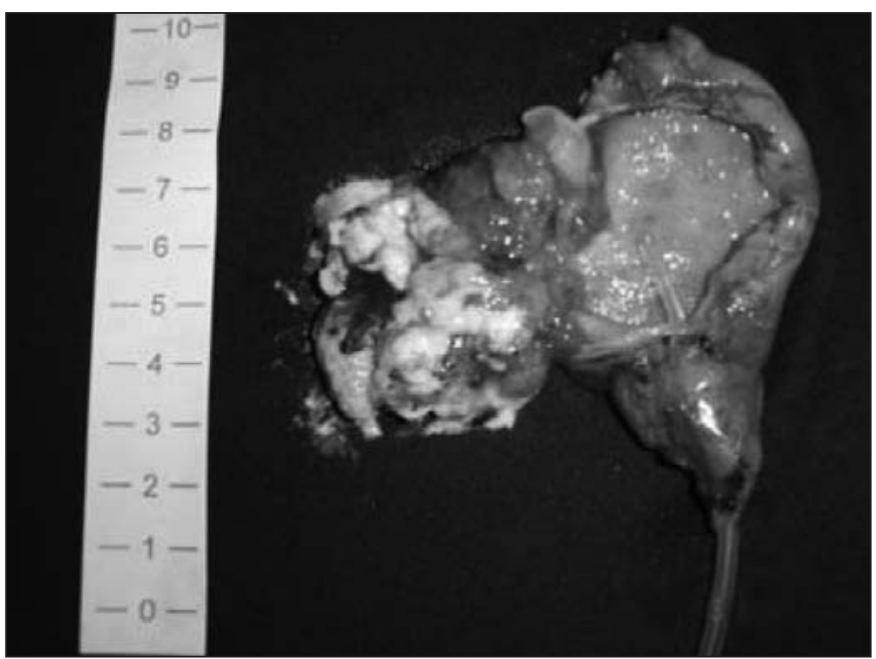

FIGURA 2. Achado macroscópico da lesão

TABELA 2. Distribuição dos achados radiológicos

\begin{tabular}{|c|c|c|c|c|c|c|c|}
\hline Características & Caso 1 & Caso 2 & Caso 3 & Caso 4 & Caso 5 & Caso 6 & Caso 7 \\
\hline Ultra-sonografia & $\begin{array}{l}2 \text { cálculos } \\
\text { de } 30 \text { e } 40 \mathrm{~mm}+ \\
\text { massa vesícula }\end{array}$ & $\begin{array}{l}5 \text { cálculos de } 5 \text { a } 20 \mathrm{~mm} \\
\text { + massa vesícula }\end{array}$ & $\begin{array}{l}10 \text { cálculos de } 5 \text { a } 30 \mathrm{~mm} \\
\text { + massa vesícula }\end{array}$ & $\begin{array}{l}2 \text { cálculos de } 20 \\
\text { e } 40 \mathrm{~mm}+\text { lesão } \\
\text { vegetante }\end{array}$ & $\begin{array}{l}3 \text { cálculos de } 5 \text { a } \\
20 \mathrm{~mm}\end{array}$ & $\begin{array}{l}3 \text { cálculos de } 5 \text { a } 15 \mathrm{~mm} \\
\text { coledocolitíase dilatação } \\
\text { via biliar }\end{array}$ & $\begin{array}{l}5 \text { cálculos de } \\
3 \text { a } 8 \mathrm{~mm}\end{array}$ \\
\hline $\begin{array}{l}\text { Tomografia / } \\
\text { ressonância }\end{array}$ & $\begin{array}{l}\text { massa } \\
\text { heterogênea }\end{array}$ & $\begin{array}{l}\text { massa } \\
\text { heterogênea }\end{array}$ & $\begin{array}{l}\text { lesão } \\
\text { vegetante }\end{array}$ & $\begin{array}{l}\text { lesão } \\
\text { vegetante }\end{array}$ & $\begin{array}{l}\text { espessamento } \\
\text { focal + calcificação }\end{array}$ & $\begin{array}{l}\text { dilatação da via biliar } \\
\text { intra e extra-hepática }\end{array}$ & $\begin{array}{l}\text { espessamento } \\
\text { difuso }\end{array}$ \\
\hline Local (lesão) & fundo & corpo & fundo & corpo & fundo & - & - \\
\hline Tamanho & $3 \mathrm{~cm}$ & $6 \mathrm{~cm}$ & $2 \mathrm{~cm}$ & $4 \mathrm{~cm}$ & $3 \mathrm{~cm}$ & - & - \\
\hline $\begin{array}{l}\text { Estádio } \mathrm{T} \text { - estimado } \\
\text { pré-operatório }\end{array}$ & T3 & T3 & T2 & T2 & T3 & ? & ? \\
\hline
\end{tabular}

TABELA 3. Distribuição dos marcadores e exames bioquímicos

\begin{tabular}{|c|c|c|c|c|c|c|c|}
\hline & Caso 1 & Caso 2 & Caso 3 & Caso 4 & Caso 5 & Caso 6 & Caso 7 \\
\hline CEA & 100 & 500 & 35 & 2,5 & 85 & 3,5 & - \\
\hline CA 19-9 & 190 & 325 & 98 & 28 & 183 & 25 & - \\
\hline Fosfatase alcalina & 500 & 750 & 33 & 10 & 25 & 38 & 10 \\
\hline Bilirrubinas totais & 1,6 & 2,0 & 1,0 & 0,6 & 0,8 & 1,0 & 0,7 \\
\hline
\end{tabular}




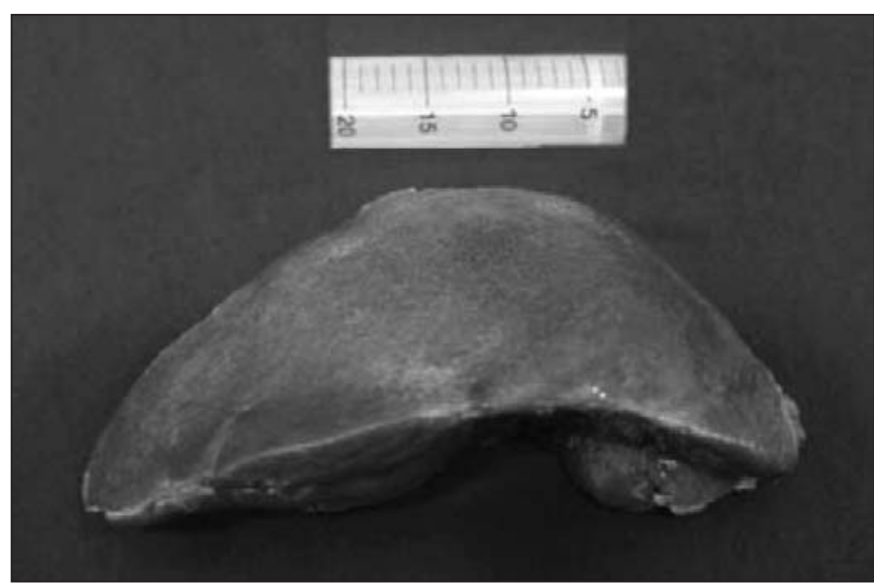

FIGURA 3. Produto da bissegmentectomia IV-B +V

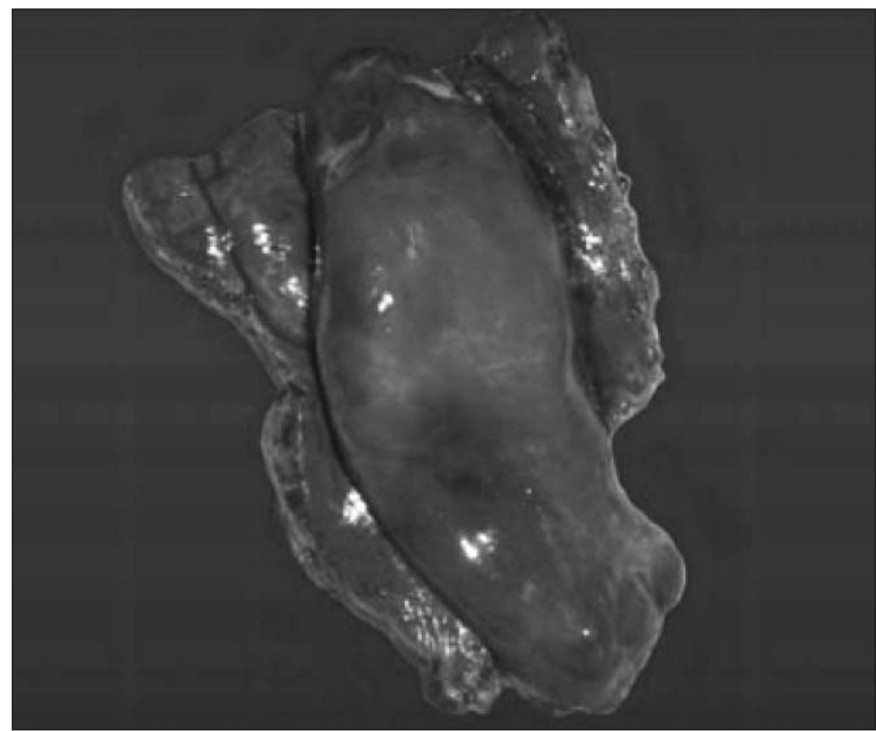

FIGURA 4. Produto de bissegmentectomia IV + V com a vesícula biliar em monobloco

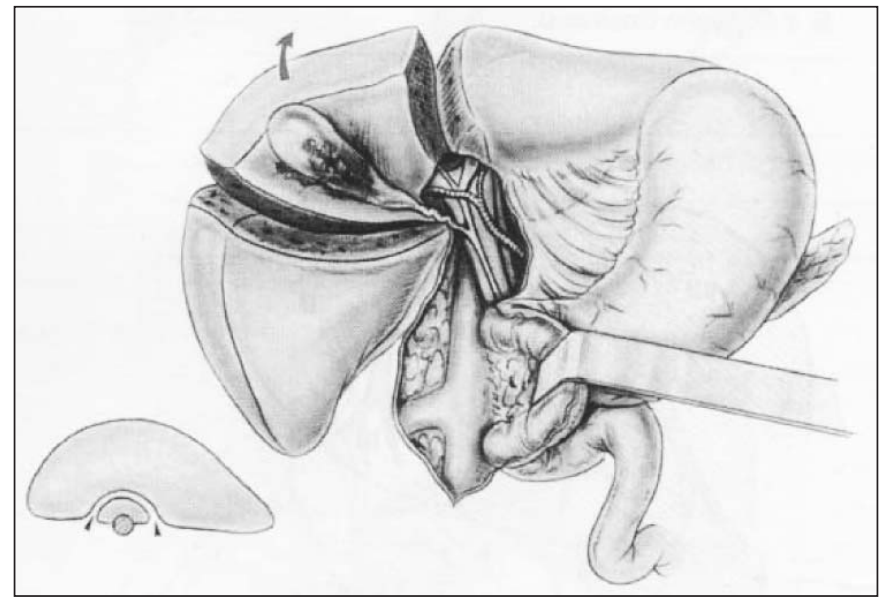

FIGURA 5. Técnica operatória da bissegmentectomia IV-B + V CUBERTAFOND et al. ${ }^{(4)}$ (com permissão)
A bissegmentectomia central inferior tem sido realizada seguindo a padronização técnica descrita por CUBERTAFOND et al. ${ }^{(4)}$ (Figura 5). Todas as ressecções hepáticas têm sido complementadas com linfadenectomia ao longo do ligamento hepatoduodenal (N1) conjuntamente à dissecção dos linfonodos N2 como pancreático-duodenais (superior, anterior, posterior), retroportais, da artéria hepática comum. A secção hepática tem sido realizada em combinação com o clampeamento hilar - manobra de Pringle (15 por 5 minutos). Na presente casuística, dois pacientes foram submetidos a colecistectomia e hepatectomia segmentar em monobloco. Enquanto em cinco pacientes foi realizada, inicialmente, a colecistectomia e após confirmação diagnóstica, seja por exame de congelação ou parafina, ressecção hepática incluindo os segmentos IV-B e $\mathrm{V}$ foi conduzida. Não tem sido realizada de forma rotineira e sistemática, a ressecção da via biliar principal. Essa conduta tem sido reservada somente para os casos em que a neoplasia comprometa o hepatocolédoco propriamente dito ou que a margem do ducto cístico tenda a ser duvidosa ou mesmo comprometida. Assim sendo, em dois pacientes cuja margem do cístico foi comprometida, a ressecção conjunta da via biliar principal (até inclusive a confluência dos ductos hepáticos) e do lobo caudado foi realizada. A reconstrução utilizada em ambos os casos foi por meio de uma hepaticojejunostomia intrahepática término-lateral de $70 \mathrm{~cm}$ em alça exclusa, retrocólica, segundo preconizado por BLUMGART e FONG ${ }^{(2)}$.

Após o tratamento cirúrgico, todos os pacientes foram seguidos com intervalos de 3 meses (até 2 anos) e de 6 meses (entre 2 e 5 anos). Em cada retorno, foram realizadas, além do exame físico completo, as dosagens de CEA e CA 19-9, US total de abdome, TC de abdome-pelve e raios- $\mathrm{X}$ simples de tórax. Recurrência foi considerada somente em casos de documentação radiológica inequívoca e, se possível, com confirmação histológica.

\section{RESULTADOS}

O tempo cirúrgico variou de 180 a 340 minutos, com média de 249 minutos. O sangramento estimado no período intra-operatório variou de $200 \mathrm{~mL}$ a $1500 \mathrm{~mL}$, com média de $757 \mathrm{~mL}$. Dois pacientes receberam transfusão de concentrado de glóbulos. O tempo de permanência hospitalar variou de 7 a 18 dias, com média de 11 dias. Nenhum paciente realizou tratamento multidisciplinar com radioterapia ou quimioterapia.

Não houve mortalidade pós-operatória. Dois pacientes apresentaram uma complicação maior e não houve complicações menores. Nenhum paciente foi reoperado. A morbidade global da presente série foi de $14,28 \%$. Um paciente submetido a bissegmentectomia ampliada, com ressecção do lobo caudado e da via biliar principal, apresentou fístula biliar de alto débito, que se resolveu com tratamento conservador. Outro paciente com cirrose micronodular de etiologia alcoólica compensada (Child A) apresentou insuficiência hepática revertida clinicamente. Essas características estão demonstradas na Tabela 4. 
Seis ressecções apresentaram margens livres (R0), enquanto uma apresentou margem microscopicamente comprometida (R1). Essa ressecção R1, foi realizada em um tumor de cerca de $6 \mathrm{~cm}$ (no maior eixo) localizado no corpo com invasão do infundíbulo (margem hepática inferior microscopicamente comprometida). Macroscopicamente, os tumores encontrados foram: papilífero $(\mathrm{n}=3)$, tubular $(\mathrm{n}=2)$ e nodular $(\mathrm{n}=2)$. Em relação ao tipo histológico do tumor, todos os pacientes apresentavam adenocarcinoma. A distribuição em relação ao grau de diferenciação do tumor foi: bem diferenciado $(n=2)$, moderadamente diferenciado $(n=3)$ e pouco diferenciado $(n=2)$. Dos sete casos operados, seis apresentavam disseminação para o leito hepático sendo classificados como "liver-bed type" enquanto apenas um caso apresentava disseminação para o hilo hepático, sendo classificado por sua vez como "hilumtype"(21). Em relação ao estádio TNM propriamente dito ${ }^{(19)}$, a distribuição encontrada foi: T2N0M0 $(n=2)$, T2N1M0 $(n=1)$, T3N0M0 $(n=3)$ e T3N1M0 $(n=1)$. A mediana de linfonodos dissecados foi de 10, variando entre 7 e 15 . Nos dois pacientes com comprometimento linfonodal ambos eram pericoledocianos; o número de linfonodos variou de um a três. Esses resultados estão demonstrados na Tabela 5.

O tempo de seguimento variou de 3 a 30 meses, com média de 16,85 meses. Apenas um paciente apresentou recidiva, e coincidentemente, apresentava margem de secção hepática microscopicamente positiva e também três linfonodos comprometidos; desenvolveu recidiva tanto no peritônio quanto no fígado em um período de 8 meses após a operação. Evoluiu com síndrome de obstrução antropilórica, foi submetido a cirurgia e acabou evoluindo para óbito no período pós-operatório (aos 9 meses de seguimento). Os demais continuam vivos, sem doença, em período de seguimento de 3 a 30 meses. Esses resultados estão demonstrados na Tabela 6 .

\section{DISCUSSÃO}

Embora a causa do carcinoma da vesícula biliar seja desconhecida, a presença de cálculos biliares tem sido considerada fator de risco para o desenvolvimento dessa neoplasia ${ }^{(39)}$. A maior evidência a favor dessa associação reside no fato de que $65 \%$ até $90 \%$ dos pacientes com neoplasia de vesícula biliar apresentem cálculos biliares por ocasião de seu diagnóstico ${ }^{(20)}$. Além disso, o risco de se desenvolver essa neoplasia se eleva de maneira diretamente proporcional ao tamanho dos cálculos. Segundo DIEHL ${ }^{(7)}$, o risco relativo para câncer de vesícula em indivíduos que tenham cálculos biliares entre 2,0 até $2.9 \mathrm{~cm}$ de diâmetro é de 2,4 frente à população geral e se eleva para 10,1 caso os cálculos apresentem diâmetro maior que $3 \mathrm{~cm}$. Na presente casuística, todos os pacientes apresentaram cálculos com diâmetros que variaram entre 0,3 a $4 \mathrm{~cm}$ de diâmetro, sendo que cinco deles mostraram cálculos maiores que $2 \mathrm{~cm}$. Embora a amostra tenha sido pequena, esse achado coincide com a supracitada teoria de que cálculos grandes podem favorecer o desenvolvimento de carcinoma de vesícula.

$\mathrm{O}$ carcinoma da vesícula biliar tem sido mais comumente descrito no fundo desse órgão. A neoplasia pode eventualmente surgir como espessamento focal da parede, lesão polipóide-vegetante ou até mesmo uma placa na sua mucosa ${ }^{(35)}$. Macroscopicamente pode ser papilífero, tubular ou nodular. Os tumores papilíferos invadem menos freqüentemente o fígado e propiciam menor

TABELA 4. Distribuição dos resultados cirúrgicos precoces

\begin{tabular}{|c|c|c|c|c|c|c|c|}
\hline & Caso 1 & Caso 2 & Caso 3 & Caso 4 & Caso 5 & Caso 6 & Caso7 \\
\hline Tempo Cirúrgico(min.) & 260 & 280 & 240 & 340 & 180 & 185 & 260 \\
\hline Sangramento estimado (ml) & 200 & 1500 & 800 & 600 & 700 & 500 & 1000 \\
\hline Tempo de Internação(dias) & 9 & 18 & 8 & 7 & 10 & 10 & 8 \\
\hline Complicações & Insuficiência hepática & Fístula biliar & - & - & - & - & - \\
\hline
\end{tabular}

TABELA 5. Distribuição dos achados histológicos

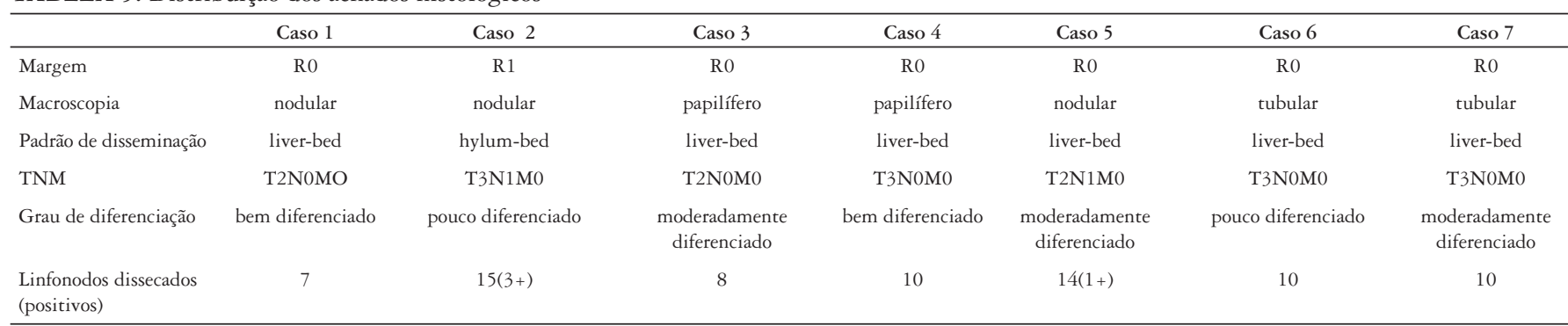

TABELA 6. Distribuição dos resultados cirúrgicos

\begin{tabular}{|c|c|c|c|c|c|c|c|}
\hline & Caso 1 & Caso 2 & Caso 3 & Caso 4 & Caso 5 & Caso 6 & Caso 7 \\
\hline Sobrevida (meses) & 20 & 9 & 9 & 19 & 30 & 3 & 28 \\
\hline Status & Vivo sem doença & Morto da doença & Vivo sem doença & Vivo sem doença & Vivo sem doença & Vivo sem doença & Vivo sem doença \\
\hline Local Recidiva & - & Peritônio + Fígado & - & - & - & - & - \\
\hline
\end{tabular}


incidência de comprometimento linfonodal ${ }^{(27)}$. Na presente série, a maioria dos pacientes apresentava tumor papilífero $(\mathrm{n}=3)$ e não havia envolvimento linfonodal em nenhum deles. Em contrapartida, os dois pacientes com o tipo nodular apresentavam comprometimento linfonodal. $\mathrm{O}$ único tipo histológico achado foi o adenocarcinoma, o que foi condizente com os achados de outros autores, em que $80 \%$ até $95 \%$ dos casos descritos têm apresentado tal histologia ${ }^{(1,12)}$.

O carcinoma de vesícula localizado não apresenta sintomas específicos. Dor em hipocôndrio direito, perda ponderal, anorexia, náuseas e vômitos, icterícia e distensão abdominal têm sido os sintomas mais comumente descritos ${ }^{(20)}$. A presença de icterícia tem sido associada a péssimo prognóstico. A irressecabilidade cirúrgica em ictéricos tem se situado em torno de 44\%. Pacientes com doença avançada podem apresentar, também, massa palpável, fígado irregular e ascite ${ }^{(20)}$.

Em 1\% dos pacientes, o carcinoma de vesícula biliar é descoberto incidentalmente durante colecistectomia de rotina ou mesmo posteriormente com a avaliação histológica da peça operatória. Contudo, somente em $20 \%$ de todos casos, a neoplasia é restrita à vesícula, sendo que a maioria se apresenta por ocasião do diagnóstico com invasão de órgãos adjacentes ou metástases à distância. A neoplasia de vesícula biliar tem que ser suspeitada em pacientes com longa história de colecistite crônica calculosa oligossintomática que comecem a apresentar mudança dos sintomas em passado recente. Mais de $90 \%$ dos pacientes têm mais de 50 anos e o pico de incidência é entre os 70 e 75 anos. A maioria dos enfermos tem sido do sexo feminino, em proporção de 3 para 1 em relação ao sexo masculino. Na presente amostra, em que pese pequena, houve predominância do sexo feminino em proporção inclusive maior de 6 para 1 . Contudo, a média etária de 66 anos foi inferior à encontrada na literatura ${ }^{(20)}$.

O conhecimento da história natural do carcinoma da vesícula biliar, em especial do seu modo de disseminação, tem sido fundamental para o desenvolvimento da sua abordagem terapêutica. FAHIM et al. ${ }^{(9)}$ descreveram os modos de disseminação dessa neoplasia: contigüidade, linfática, hematogênica, neural, peritonial e intraductal. A vesícula biliar tem sua drenagem linfática direcionada, primariamente para os linfonodos ao longo do sistema biliar principal, que correspondem ao seu primeiro nível de drenagem (N1). A drenagem subseqüente ocorre para os linfonodos pancreático-duodenais (superior, anterior e posterior), da veia porta (posterior) e ao longo da artéria hepática comum que são, por sua vez, considerados segundo nível de drenagem (N2). Finalmente a drenagem linfática segue para os linfonodos do tronco celíaco, vasos mesentéricos superiores e para-aórticos que correspondem ao terceiro nível de drenagem (N3). Linfonodos hilares somente são comprometidos por drenagem retrógrada. O índice de envolvimento linfonodal depende do tipo macroscópico do tumor, da profundidade de sua invasão e do grau de diferenciação, e tem se situado em geral, de $54 \%$ até $64 \%{ }^{(20)}$. Para tumores $\mathrm{T} 1 \mathrm{~b}$, situa-se em torno de $15,5 \%$ para cadeias primárias (N1), crescendo para $40 \%$ até $60 \%$ para cadeias primárias e cerca de $20 \%$ para cadeias secundárias nos tumores T2(21).
Já em tumores T3 ou T4, o comprometimento de linfonodos para-aórticos (N3) pode alcançar índices em torno de 19\% até $25 \%(26,33,38)$.

Devido à disseminação precoce tanto linfática quanto peritonial, a minoria dos pacientes, por ocasião de seu diagnóstico, apresenta doença localizada passível de ressecção curativa. E por se tratar de doença mais comumente observada nas populações idosas, poucos enfermos têm condições clínicas ideais para realização de operações extensas e complexas. Por ocasião do diagnóstico, a maioria dos doentes se encontra em estádios avançados, com sintomatologia intensa e baixo índice de ressecabilidade ${ }^{(21,28)}$.

Embora o diagnóstico do carcinoma da vesícula biliar seja histológico (anátomo-cirúrgico), ele deve ser sempre aventado frente a uma imagem radiológica suspeita. Destarte, pacientes com quadro clínico compatível com colelitíase (cólica biliar ou síndrome dispéptica) podem apresentar coincidentemente ao exame de imagem, lesão suspeita de câncer de vesícula e que merece melhor avaliação radiológica. A US de abdome tem sido o exame de triagem mais realizado, pois além de fácil disponibilidade e custo acessível, alia boa precisão para o diagnóstico. Como tem sido observada freqüentemente a associação entre cálculos biliares e neoplasia, os sintomas apresentados por ambas as afecções podem ser confundidos e desta maneira a US pode revelar sinais radiológicos que possam, eventualmente, surpreender uma neoplasia curável. As imagens radiológicas, tanto as da US quanto as da TC, se correlacionam fortemente com a aparência macroscópica do tumor. Têm sido descritos três padrões de apresentação: a) massa tumoral que substitui a vesícula biliar (36\%-45\%), b) espessamento da parede vesicular (19\%-47\%) e c) massa vegetante na luz da vesícula biliar $(14 \%)^{(10,29)}$. Outros autores têm reportado dois grandes grupos de achados radiológicos (US e TC). No primeiro grupo, tem sido identificada massa dentro da luz da vesícula biliar, enquanto no segundo grupo, grande massa ocupando toda fossa vesicular, inclusive substituindo totalmente a vesícula biliar. O grupo 1, por sua vez, tem sido subdividido em três tipos, de acordo com a morfologia da lesão: 1) massa ocupando a maior parte da luz da vesícula, 2) massa polipóide projetando-se para seu interior e 3) tumoração infiltrante, apresentado-se como um espessamento focal ou difuso da parede ${ }^{(16,17)}$. A forma mais comumente descrita de carcinoma de vesícula biliar tem sido de uma massa que a substitui. Esse tipo de massa tem sido observado como uma tumoração heterogênea, que ocupa o espaço subhepático e que pode se apresentar com cálculos em seu interior, indicando assim, a origem vesicular da lesão. Embora sejam as apresentações menos comuns, os tumores intraluminares são comumente observados como massas fungóides para o interior da luz e são altamente suspeitas de neoplasia.

Tem sido relatado que o tamanho do tumor está estritamente relacionado com a extensão tumoral por ocasião do diagnóstico. Os tumores com mais de $1 \mathrm{~cm}$ são mais provavelmente malignos, enquanto os menores que $1 \mathrm{~cm}$ são, a princípio, benignos e comumente relacionados a pólipos de colesterol $^{(18)}$. O espessamento da parede da vesícula, tanto focal quanto difuso, 
tem sido a forma mais difícil de estabelecer diagnóstico de câncer de vesícula biliar, pois pode ser indistinguível de colecistite aguda. No entanto, a presença conjunta de irregularidade e a descontinuidade da mucosa, aliadas a um espessamento da parede (local ou difuso), pode ser mais freqüentemente encontrada em carcinoma de vesícula ${ }^{(41)}$.

$\mathrm{Na}$ presente série, a maioria dos pacientes apresentava lesão polipóide ou massa na fossa vesicular (80\%), o que é condizente com o observado na literatura. Apenas uma lesão se apresentava com espessamento focal e calcificação. A RNM tem sido a modalidade mais sensível para detecção da extensão do tumor. Através dela pode ser determinada com boa precisão a invasão direta do fígado ou de estruturas circunvizinhas, além de possibilitar a avaliação de estruturas vasculares e da via biliar principal e secundária. Portanto, pode ser de fundamental importância para a determinação pré-operatória da ressecabilidade ${ }^{(29)}$. Em que pese toda a evolução das técnicas de imagem, a minoria desses tumores tem sido detectada no período pré-operatório, o que infelizmente tem acarretado diagnósticos tardios e em estádios avançados, com baixo índice de curabilidade ${ }^{(2,3)}$. Na presente casuística, o diagnóstico préoperatório foi de fundamental importância para um planejamento minucioso do tipo de ressecção a ser planejada.

Ambos os marcadores CA 19-9 e CEA podem estar elevados no carcinoma de vesícula e, ocasionalmente, têm contribuído para o diagnóstico, mormente nos pacientes anictéricos ${ }^{(13)}$. Segundo De ARECTXABALA et al. ${ }^{(5)}$, esses marcadores têm apresentado sensibilidade similar (em torno de 70\%). No entanto, a especificidade do CA19-9 tem sido maior que a do CEA (90\% contra 71\%). Na presente série, ambos os marcadores estiveram elevados em $80 \%$ dos pacientes (quatro de cinco indivíduos dosados no pré-operatório) e todos se apresentavam anictéricos. Apesar da pequena casuística relatada, esse achado sinaliza que associação da imagem suspeita com marcadores elevados em pacientes anictéricos, talvez possa contribuir para o diagnóstico de carcinoma de vesícula. Segundo NOSHIRO et al. (24), além da contribuição diagnóstica, a elevação desses marcadores na presença de carcinoma de vesícula também pode estar relacionada com seu prognóstico.

O tratamento do carcinoma da vesícula biliar ainda é motivo de desafio e controvérsia para o cirurgião. Se por um lado a cirurgia alargada com hepatectomia e linfadenectomia regional pode levar ao prolongamento da sobrevida, por outro, ainda

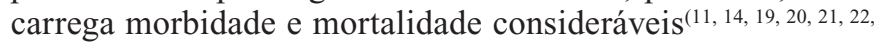
$23,24,25,30,31,37,39)$. A cirurgia deve ser individualizada para cada caso; tanto a hepatectomia direita ampliada para o segmento IV, quanto a bissegmentectomia central inferior (ressecção dos segmentos IV-B e V) ou ainda mesmo a hepatectomia central (ressecção dos segmentos IV, V e VIII), têm sido aceitas pela maioria dos serviços especializados, desde que respeitadas as margens que devem ser, sempre que possível, livres $^{(2,3,13,14,20,21,39)}$. Desta maneira, para os tumores T2-3 restritos ao fundo ou corpo da vesícula, sem invasão hilar ("liver-bed tumor") e, principalmente, sem crescimento infiltrativo (com pouca invasão para o fígado), a bissegmentectomia central inferior $(\mathrm{S} 4+\mathrm{S} 5)$ pode ser realizada sem alteração do resultado oncológico final. Essa operação apresenta como principal vantagem, a maior preservação do parênquima hepático em relação à hepatectomia direita e sem o comprometimento da radicalidade oncológica ${ }^{(11,19,25,37,39)}$. Essa operação parece apresentar morbidade menor que a da hepatectomia direita ampliada, principalmente em relação à insuficiência hepática pós-operatória e à hemorragia transoperatória. A morbidade de $14,28 \%$ da presente série, está dentro da relatada na literatura que tem variado de $13 \%$ a $30 \%{ }^{(19,36,37)}$. As principais complicações têm sido infecciosas, como abscesso intracavitário ou pneumonia, mas também têm sido descritos derrames pleurais e fístulas biliares. Deve-se salientar que os dois casos em que houve complicação, foram tratados conservadoramente sem a necessidade de intervenção cirúrgica. Um deles evoluiu para uma fístula biliar, complicação comum, principalmente em ressecções que envolvam o lobo caudado e confecção de anastomose biliodigestiva intra-hepática. Esta fístula foi tratada clinicamente e acabou por fechar espontaneamente no $30^{\circ}$ dia pós-operatório. A outra complicação — uma insuficiência hepática transitória - foi também resolvida com tratamento conservador (clínico). O paciente apresentava hepatopatia não suspeitada no pré-operatório, que estava clinicamente compensada. Tem sido reconhecido que, mesmo ressecções hepáticas menores estão associadas à insuficiência hepática pós-operatória nesse tipo de doente. Apesar da morbidade, a mortalidade da bissegmentectomia central inferior tem sido baixa. Embora faltem estudos comparativos entre as duas técnicas, a mortalidade é bem inferior à observada na hepatectomia direita ampliada. Embora a presente casuística seja pequena, a mortalidade encontrada foi de $0 \%$, similar à publicada por SUZUKI et al. ${ }^{(36)}$ em 19 pacientes. Em contrapartida, a hepatectomia direita ampliada, apesar de sua grande radicalidade oncólogica, tem cursado com elevada mortalidade, mesmo em grandes centros especializados (até 30\%), principalmente no paciente idoso e ictérico ${ }^{(14,15,30)}$. Assim sendo, de acordo com alguns autores, essa operação deve ser reservada para os tumores mais avançados (T3 ou T4) e que apresentem, principalmente, localização distal (infundíbulo e região do ducto cístico), invasão hilar ("hilum-type") ou caráter infiltrativo com extensão profunda para o leito hepático ${ }^{(2,3,13,14,20,21,30)}$. Embora existam estudos que demonstrem aumento da sobrevida dos pacientes com linfonodos comprometidos submetidos a linfadenectomia alargada, para essa doença tal procedimento apresenta papel decisivo no estádio e na avaliação prognóstica, suplantando para alguns o seu caráter propriamente terapêutico. Tem sido preconizada a dissecção dos linfonodos ao longo do ligamento hepatoduodenal (N1), podendo ser estendida para as cadeias pancreato-duodenais, da artéria hepática comum, do tronco celíaco e retro-portais (N2), tendo como objetivo maior precisão do estádio ${ }^{(21,30,37,39)}$.

Controvérsia reside na ressecção (em bloco) da via biliar e do lobo caudado de maneira sistemática. Se, para alguns autores, é sempre realizada para facilitar a linfadenectomia hilar e aumentar a radicalidade cirúrgica, para outros deve ser reservada somente para os casos de invasão propriamente dita dessas estruturas ou mesmo margem insuficiente. A ressecção 
de forma sistemática não tem aumentado a sobrevida e ainda carrega maior morbidade, principalmente em relação às fistulas biliares $^{(3,13,20,21,37)}$. Na presente casuística, a ressecção da via biliar e do lobo caudado foi realizada somente nos casos em que a invasão era incontestável ou mesmo quando a margem cirúrgica se apresentava exígua em relação a essas estruturas. Dois pacientes realizaram o referido procedimento ampliado, pois apresentavam tanto margem exígua junto à via biliar principal, quanto ao lobo caudado. Mesmo com essa indicação bem consolidada, conforme ponto de vista dos autores, um deles desenvolveu complicação pós-operatória (fístula biliar).

A sobrevida observada nos pacientes com carcinoma de vesícula tem sido diretamente proporcional à espessura do tumor, à presença de comprometimento de linfonodos (estádio TNM) e à cirurgia sem margens comprometidas (R0), respectivamente. Após ressecção curativa (R0), a sobrevida tem variado de $60 \%$ a $80 \%$ em 5 anos para os tumores T2, e de $15 \%$ a $50 \%$ em 5 anos para os tumores $\mathrm{T}^{(21)}$. Apenas uma cirurgia apresentou margem comprometida (R1), ou seja, 86\% delas foram R0, consolidando desta maneira, essa operação em relação a sua radicalidade oncológica, desde que seguida à risca sua indicação (tumor do fundo/corpo, tipo papilífero ou nodular, sem invasão hilar ou hepática extensa). Não obstante o período de seguimento tenha sido curto, apenas um paciente apresentou recidiva e faleceu (cirurgia com margem microscópica comprometida). Todos os demais estão vivos em período de seguimento que variou de 3 a 30 meses.

\section{CONCLUSÃO}

A bissegmentectomia central inferior $(\mathrm{S} 4 \mathrm{~B}+\mathrm{S} 5)$ é uma opção oncologicamente segura para o tratamento cirúrgico do carcinoma de vesícula em casos selecionados (tumores T2 ou T3 do fundo/corpo da vesícula e do tipo "liver-bed", principalmente nos seus padrões nodular ou papilífero de disseminação). Essa operação tem apresentado considerável morbidade, contudo sua mortalidade tem sido baixa.

Costa SRP, Horta SHC, Miotto MJ, Costas MC, Godinho CA, Henriques AC. Central inferior bisegmentectomy (S4B+S5) for gallbladder carcinoma treatment: a series of seven resectable cases. Arq Gastroenterol. 2008;45(1):73-81.

ABSTRACT - Background - Despite its rarity, gallbladder cancer is an aggressive type of neoplasia with a very poor prognosis. The best resection for oncological purposes continues to be right hepatectomy extended to segment IV. However, bisegmentectomy IV-V is becoming an interesting alternative because of greater preservation of the parenchyma. Aim - To report the early and late results from bisegmentectomy IV-V in cases of carcinoma of the gallbladder. Methods - A series of seven cases of invasive carcinoma is presented (six women and one man). These patients underwent bisegmentectomy IV-V at the General Surgery Service of the Teaching Hospital of the ABC Medical School, Santo André, SP, Brazil. The study was conducted between 2002 and 2006 . The patients' ages ranged from 52 to 72 years. The diagnosis was preoperative (radiological) in five cases, which were all confirmed by intraoperative frozen-tissue examination, while in two cases the diagnosis was postoperative, following open cholecystectomy. Results - The duration of the operation ranged from 180 to 340 minutes. The quantity of intraoperative bleeding ranged from 200 to $1500 \mathrm{~mL}$. There were two major complications but no mortality. Six patients did not present any recurrence over the course of 3 to 30 months of follow-up. Conclusion - Bisegmentectomy IV-V may constitute a curative surgical alternative for treating gallbladder cancer. This procedure presents acceptable morbidity and mortality.

HEADINGS - Gallbladder neoplasms. Carcinoma, surgery. Hepatectomy. 


\section{REFERÊNCIAS}

1. Albores-Saavedra J, Henson DE, Sobin LH The WHO histological classification of tumours of the gallbladder and extrahepatic bile duct. A commentary on the second edition. Cancer. 1992;70:410-4

2. Blumgart LH, Fong Y. Cancer of the bile ducts and common bile duct. In: Surgery of the liver and biliary tract. $3^{\text {th }}$ ed. Edinburgh: WB Saunders; 2000. 41p.

3. Choi EA, Rodgers SE, Ahmad SA, Abdalla EK. Hepatobiliary cancers. In: Feig BW, Berger DH, Fuhrman GM, editors. The M.D. Anderson surgical oncology handbook. 4th ed. Philadelphia: Lippincott Williams \& Wikins; 2006. p.320-66.

4. Cubertafond P, Launois B, Gainant A, Malier F. Traitment chirurgical des cancer de la voie biliare. In: Encyclopédie médico-chirurgicale. Techniques chirurgicales - Appareil digestif. Paris: Édition techniques; 1990. 40-970.

5. De Arectxabala X, Riedman JP, Roa I, Wentzel C, Inostrosa J, Burgos L. Ca 19-9 and carnoembryonic antigen in gallbladder cancer. Rev Med Chile. 1996;124:11-20.

6. Destoll M. Rationis mendenchi. In: Batavarum L, editor. Nosocomio practico vendonbenensi - Part 1. Honkoop, Hoak et Socios et A et J; 1788.

7. Diehl AK. Gallstone size and the risk of gallbladder cancer. JAMA. 1983;250:2323-6.

8. Donohue JH, Stewart AK, Menck Hr. The national cancer data base report on carcinoma of the gallbladder, 1989-1995. Cancer 1998;83:2618-28.

9. Fahim RB, MacDonald JR, Richards JC, Ferris DO. Carcinoma of the gallbladder: a study of its modes of spread. Ann Surg. 1962;156:114-24.

10. Franquet T, Montes M, Ruiz DE, Azúa Y. Primary gallbladder carcinoma: imaging findings in 50 patients with pathologic correlation. Gastrointest Radiol. 1991;16:143-8.

11. Gatica FM. Cáncer de la vesicula biliar. Resultados de la cirurgia resectiva. Ver Chil Cir. 2002;54:464-73.

12. Henson DE, Albores-Saavedra J, Corle D. Carcinoma of the gallbladder. Histological types, stage of disease, grade, and survival rates. Cancer. 1992:70:1493-7.

13. Koea JB, Blumgart LH. Câncer de vesícula. In: Correia MC, Mello ELR, Santos CER, editores. Cirurgia do Câncer Hepatobiliar. Revinter. 2003:124-37.

14. Kondo $\mathrm{S}, \mathrm{Katoh} \mathrm{H}$. Indication and operative techniques of extended right hepatic lobectomy for advanced cancer Nippon Geka Gakkai Zasshi. 2002;103·549-52.

15. Kondo S, Nimura Y, Hayakawa N, Kamiya J, Nagino M, Uesaka K. Extensive surgery for carcinoma of the gallbladder. Br J Surg. 2002;89:179-84

16. Kumar A, Aggarwal S, Bery M. Ultrasonography of carcinoma of gallbladder: an analysis of 80 cases. J Clin Ultrasound. 1990;18: 715-20.

17. Kumar A, Aggarwal S. Carcinoma of the gallbladder: CT findings in 50 cases. Abdom Imaging. 1994;19:304-8

18. Lane J, Buck JL, Zeman RK. Primary carcinoma of the gallbladder: a pictorial essay. Radiographics. 1989;9;209-28.

19. Manterola C, Gutierrez R, Bustos L, Vial M, Muñoz S, Pineda V, Fernandez O. Experiencia y resultados del tratamiento de pacientes com cáncer de la vesicula biliar. Rev Chil Cir. 2002;54:605-10

20. Misra S, Chatuverdi A, Misra NC, Sharma ID. Carcinoma of the gallbladder. Lancet Oncol. 2003;4:167-76.

21. Muratore A, Polastri R, Capussotti L. Radical surgery for gallbladder cancer: current options. Eur J Surg Oncol. 2000;26:438-43.

22. Myiazaki M, Itoh H, Ambiru S, Shimizu H, Togawa A, Gohchi E, Nakajima N. Radical surgery for advanced gallbladder carcinoma. Br J Surg. 1996;83:478-81.

23. Nakamura S, Sagakuchi S, Susuki S, Muro H. Aggressive surgery for carcinoma of gallbladder. Surgery. 1989;106:467-73
24. Noshiro H, Chijiwa K, Yamaguchi K, Shimizu S, Sugitani A, Tanaka M. Factors affecting surgical outcome for gallbladder carcinoma. Hepagastroenterology. 2003;50:939-44.

25. Ogura Y, Mizumoto Isaji S, Kusuda T, Matsuda S, Tabata M. Radical operations for carcinoma of gallbladder. World J Surg. 1991;15;337-43.

26. Ogura Y, Tabata M, Kawarada Y, Mizumoto R. Effect of hepatic invasion on the choice of hepatic resection for advanced carcinoma of the gallbladder: histologic analysis of 32 surgical cases. World J Surg. 1998;22:262-7.

27. Onoyama H, Yamamoto M, Tseng A, Ajiki T, Saito Y. Extended cholecistectomy for carcinoma of the gallbladder. World J Surg. 1995;19:758-63.

28. Puhalla H, Wild T, Bareck E, Pokorny H, Ploner M, Soliman T, Stremitzer S, Depisch D, Laengle F, Gruenberger T. Long-term follow-up of surgically treated gallbladder cancer patients. Eur J Surg Oncol. 2002;28:857-63.

29. Rooholamini SA, Tebrani NS, Razari MK. Emerging of gallbladder carcinoma Radiographics. 1994;14:291-306.

30. Sasaki R, Takahashi M, Funato O, Nitta H, Murakami M, Kawamura H, Suto T, Kanno $\mathrm{S}$, Saito K. Hepatopancreatoduodenectomy with wide lymph node dissection for locally advanced carcinoma of the gallbladder - Long term results. Hepatogastroenterology. 2002;49:912-5.

31. Sasaki R, Takahashi M, Funato O, Nitta H, Murakami M, Kawamura H, Suto T, Kanno $\mathrm{S}$, Saito K. Long-term results of central inferior (S 4a + S5) hepatic subsegmentectomy and pancreatoduodenectomy combined with extended lymphadenectomy for gallbladder carcinoma with suserous or mild invasion (pT2-3) and nodal involvement: a preliminary report. Hepatogastroenterology. 2004;51:215-8.

32. Sheinfield W. Cholecystecthomy and partial hepatectomy for carcinoma of the gallbladder with local liver extension. Surgery. 1947;22:48-58.

33. Shimada H, Endo I, Togo S, Nakano A, Izumi T, Nakagawara G. The role of lymph node dissection in the treatment of gallbladder carcinoma. Cancer 1997:79:892-9.

34. Sobin LH, Wittekind Ch. Digestive system: gallbladder. In: TNM classification of malignant tumors. $6^{\text {th }}$ ed. New York: Wiley-Liss; 2002. p.84-6.

35. Sumiyoshi K, Nagai E, Chijiiwa K, Nakayama F. Pathology of carcinoma of the gallbladder. World J Surg. 1991;15:315-21.

36. Suzuki S, Yokoi Y, Kurachi K, Inaba K, Ota S, Azuma M, Konno H, Baba S, Nakamura S. Appraisal of surgical treatment for pT2 gallbladder carcinomas. World J Surg. 2004;28:160-5.

37. Taner CB, Nagorney DM, Donohue JH. Surgical treatment of gallbladder cancer. J Gastrointest Surg. 2004;8:83-9.

38. Tsukada K, Kurosaki I, Uchida K. Lymph node spread from carcinoma of the gallbladder. Cancer. 1997;80:661-7.

39. Varshney S, Buttirini G, Gupta R. Incidental carcinoma of the gallbladder. Eur J Surg Oncol. 2002;28:4-10

40. Zatonsky WA, Lowenfels AB, Boyle P. Epidemiologic aspects of gallbladder cancer: a case control study of the SEARCH program of the International Agency for Research on Cancer. J Natl Cancer Inst. 1997;89:1132-8.

41. Wibbenmeyer LA, Sharafuddin MJ, Wolverson MK. Sonographic diagnosis of unsuspected gallbladder cancer: imaging findings in comparison with benign gallbladder conditions. AJR. 1995;165:1169-74. 